\title{
AVALIAÇ̃̃o DA CURVATURA DO BOCAL DE UM TÚNEL DE VENTO SUBSÔNICO E ABERTO, UTILIZANDO SIMULAÇÃO NUMÉRICA
}

\author{
Rafael Silva Araripe e Francisco Frederico dos Santos Matos \\ Instituto Federal de Educação, Ciência e Tecnologia do Ceará, Campus de Maracanaú \\ Programa de Pós-graduação em Energias Renováveis (PPGER) \\ <rafasararipe@gmail.com>, <francisco.f.matos@gmail.com>
}

DOI: 10.21439/conexoes.v10i5.1170

\begin{abstract}
Resumo. O presente trabalho tem como objetivo avaliar numericamente um túnel de vento aberto e subsônico, a ser usado na calibração de anemômetros de copos, que são amplamente utilizados na indústria de parques eólicos. Através da solução numérica das equações de Navier-Stokes para o escoamento que ocorre no interior do túnel, esse estudo preliminar trata da definição da curva de contração a ser utilizada na concepção do bocal de entrada. Tal curvatura influencia diretamente na qualidade do fluxo que segue para a seção de testes, além de interferir no comprimento necessário para se atingir as velocidades demandadas na calibração de anemômetros. Através parâmetros pré-estabelecidos, foram comparadas 5 curvas de Bezier, onde os efeitos sob o deslocamento do ar direcionado à seção de testes foram avaliados. Como resultado, foi possível verificar a influência causada pela curva de contração perante as diferentes condições geométricas apresentadas.
\end{abstract}

Palavras-chaves: Túnel de Vento Subsônico Aberto. Bocal de Entrada. Calibração de Anemômetros.

\begin{abstract}
This study aims to numerically evaluate an open and subsonic wind tunnel, to be used in calibration of cup anemometers, which are widely used in wind farm industry. Through the numerical solution of the Navier-Stokes equations for the flow that occurs inside the tunnel, this preliminary study addresses the contraction curve definition to be used in the inlet nozzle design. Such curvature directly influences the quality of the flow that goes into the test section, and interfere with the length needed to reach the speeds demanded in anemometers calibration. Through pre-established parameters five Bezier curves were compared, where the effects on the air displacement directed to the test section were evaluated. As the result, it was possible to verify the influence caused by the contraction curve on the different geometric conditions presented.
\end{abstract}

Keywords: Open Subsonic Wind Tunnel. Inlet nozzle. Calibration of Anemometers.

\section{INTRODUÇÃO}

Tendo em vista a crescente demanda por fontes renováveis de energia elétrica, a energia eólica tem atraído muitos investimentos e pesquisas para o setor. Na instalação de um novo parque eólico, são efeituadas verificações do potencial energético da região, que consiste na avaliação das velocidades do vento através da utilização de anemômetros. Para garantir a veracidade das medições e assim evitar desperdício dos recursos financeiros, os equipamentos devem ser calibrados periodicamente utilizando um túnel de vento.

Considerando um projeto de túnel de vento aberto e fluxo subsônico para atender a demanda do estado do Ceará e da região Nordeste na calibração de anemôme- tros, devem ser levados em consideração vários aspectos relevantes no seu desenvolvimento. Dentre estes aspectos está o desenvolvimento geométrico de cada um dos 5 componentes que podemos destacar em um túnel de vento. É abordado neste artigo o estudo do bocal de entrada do fluxo de ar, que vem como primeiro componente a ser analisado. Segundo BARLOW; RAE; POPE (1999) os aspectos mais relevantes para o desenvolvimento do bocal de entrada do sistema são: a vazão de ar necessária na seção de testes para realizar a calibração; a qualidade do fluxo em relação a sua homogeneidade laminar determinada pelo número de Reynolds; e espaço disponível para construção do equipamento.

O bocal tem como função prioritária garantir a con- 
tração da massa de ar e direcioná-la para entrada da seção de teste de forma suave. Por definição, o componente possui uma entrada maior que a saída para orientar o fluído para dentro do túnel. Segundo ARRIAS et al. 2008) GROFF; ALÉ (2000) e MEHTA; BRADSHAW (1979) a razão entre a área da entrada e a área da saída e a curvatura da parede são os principais parâmetros para o desenvolvimento do componente.

Com isso, o artigo contempla a comparação entre 5 curvas de Bezier, semelhantes as curvas propostas por LÉPINE; GUIBOULT; TRÉPANIER (2001) e LEIFSSON; KOZIEL (2015), com o intuito de identificar a respectiva influência do fluxo na entrada da seção de testes. Além disso, o estudo de malha afim de estabelecer o refino mais adequado para a análise em questão. No desenvolvimento, foram consideradas as dimensões de entrada e saída em função do modelo desenvolvido por LEIFSSON; KOZIEL (2015) com o intuito de validar os resultados.

\section{OBJETIVOS}

Tendo em vista a importância e relevância do desenvolvimento do projeto do bocal para a construção do túnel de vento, este artigo tem por objetivo analisar a curvatura de suas paredes de contração. Desta forma serão desenvolvidas e comparadas cinco curvas diferentes para avaliar a influência da contração do bocal no fluxo do túnel de vento.

\section{REFERENCIAL TEÓRICO}

Para facilitar o entendimento do tema objeto deste projeto, faz-se necessária uma breve revisão sobre a classificação de túneis de vento, calibração de anemômetros e curva de Bezier.

\subsection{Classificação de Túneis de Vento}

Túneis de vento são instrumentos de pesquisa utilizados no estudo de movimentos do ar através de objetos sólidos (ARRIAS et al., 2008). Têm como objetivo promover um fluxo de ar em condições controláveis de velocidade, temperatura, pressão, umidade, etc., para se obter dados sobre os seus efeitos. Existem vários tipos e modelos de túneis de vento, e podem ser caracterizados por vários aspectos. O principal deles é quanto ao circuito, aberto ou fechado. Mas, também podem ser diferenciados quanto à velocidade de escoamento em relação à velocidade do som, quanto ao sentido do escoamento, dentre outras características (MEHTA; BRADSHAW 1979).

\subsubsection{Quanto ao circuito}

- Sistema Aberto Neste sistema o fluxo de ar é puxado da atmosfera e passa por todo o túnel até retornar à atmosfera, conforme é ilustrado na Figura 1. Vantagens: baixo custo de construção; tamanho reduzido; e pode ser usado com sistema de fumaça para melhor visualização do escoamento. Desvantagens: geralmente são barulhentos; demandam mais energia para gerar o escoamento; e apresentam dificuldades no controle da temperatura do ar.

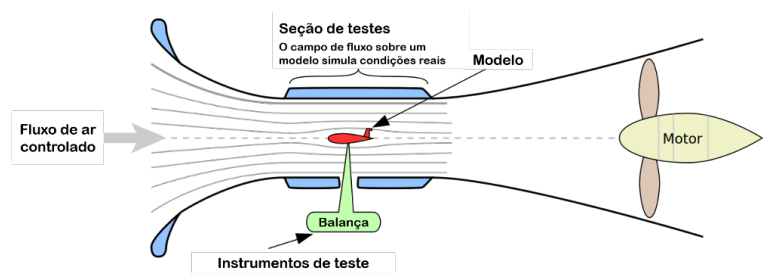

Figura 1: Túnel de vento de circuito aberto. Fonte: SULLIVAN, 2001 (adaptada).

- Sistema Fechado No sistema fechado o fluxo de ar circula dentro do túnel continuamente, através de dutos que redirecionam o escoamento que passou pela seção de teste para a entrada do sistema novamente, conforme ilustrado na Figura 2. Vantagens: demanda menos energia para geração do escoamento; mais fácil de controlar os parâmetros do escoamento (velocidade, temperatura, pressão, umidade); e melhor qualidade de fluxo. Desvantagens: maior custo de construção; e demanda de grandes espaços (MEHTA; BRADSHAW, 1979).

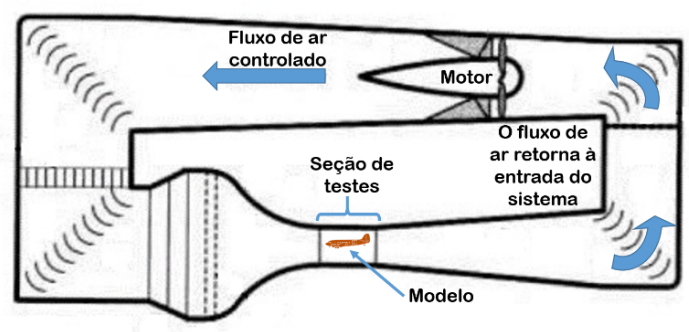

Figura 2: Túnel de vento de circuito fechado. Fonte: BARLOW et al, 1999 (adaptada).

\subsubsection{Quanto á velocidade}

Comparando com a velocidade do som $(340 \mathrm{~m} / \mathrm{s})$, existem três tipos túneis de vento: - Subsônico $(<340 \mathrm{~m} / \mathrm{s})$; - Transônico (= $340 \mathrm{~m} / \mathrm{s})$; - Supersônico (> $340 \mathrm{~m} / \mathrm{s})$. 


\subsubsection{Quanto ao sentido do escoamento}

Os túneis de vento de circuito aberto podem ser divididos em duas categorias (BARLOW; RAE; POPE 1999): - Soprador: o ventilador que promove o fluxo de ar é posicionado na entrada do equipamento e impulsiona o vento diretamente para dentro do sistema; Sugador: o exaustor que promove o fluxo de ar é posicionado na saída do sistema, extraindo o ar de dentro do túnel e forçando o fluxo de dentro para fora.

\subsection{Componentes}

Os túneis de vento abertos podem ser divididos em 5 partes básicas além do corpo a ser analisado conforme Figura 3 abaixo:

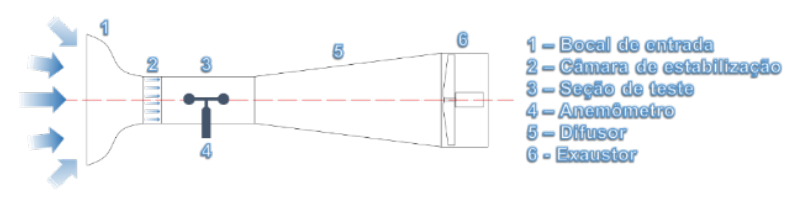

Figura 3: Partes do túnel de vento.

\subsubsection{Bocal de entrada}

O bocal de entrada do túnel de vento tem a função de facilitar a captação do ar externo, direcioná-lo para câmara de estabilização e aumentar a energia cinética do escoamento com a perda de pressão através da contração, Segundo GROFF; ALÉ (2000).

\subsection{Calibração de Anemômetros}

No Brasil, a EPE (Empresa de Pesquisa Elétrica), vinculada ao MME (Ministério de Minas e Energia), solicita um estudo de certificação de vento para habilitação técnicas de empreendimentos eólicos, conforme mencionado por (ALÉ et al. 2009). Esta certificação é realizada através do estudo do potencial eólico da região utilizando-se de anemômetros para medir a intensidade e direção dos ventos. Para a liberação desta habilitação aos empreendimentos, é exigido que o levantamento de dados seja realizado utilizando-se de anemômetros com certificado de calibração. A calibração de anemômetros é regulamentada mundialmente pela MEASNET (2009), que por sua vez define os parâmetros do fluxo de ar utilizado para calibração do equipamento em questão (Figura 4).

O processo deve ser realizado em um túnel de vento com capacidade de proporcionar um fluxo de ar com parâmetros predeterminados e comparar com os dados fornecidos pelo anemômetro durante os testes. Tendo

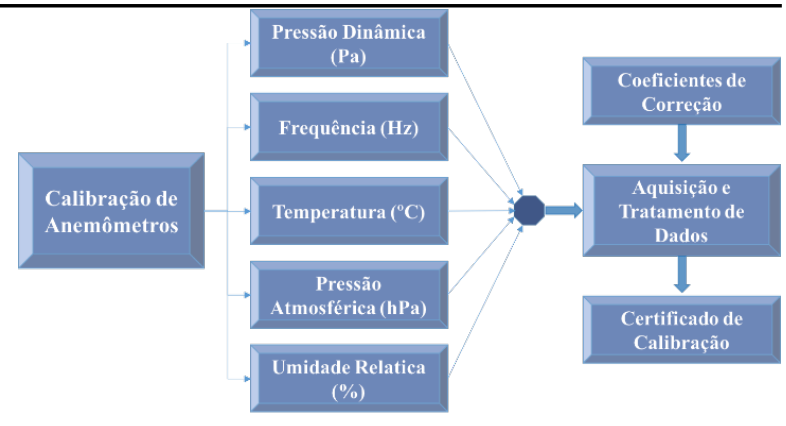

Figura 4: Parâmetros para calibração de anemômetros

como base as especificações fornecidas pela MEASNET (2009), o teste consiste em variar a velocidade do escoamento de $4 \mathrm{~m} / \mathrm{s}$ a $16 \mathrm{~m} / \mathrm{s}$, colher os dados do anemômetros para cada faixa de velocidade com uma frequência de amostragem de pelo menos $1 \mathrm{~Hz}$ durante 30 segundo no mínimo, tratar os dados fornecidos pelo anemômetro com os coeficientes de correção predeterminados para o túnel de vento, e gerar o certificado de calibração. Para que o procedimento de calibração seja considerado satisfatório, o coeficiente de correlação deve ser maior que 0,99995 .

\subsection{Curva de Bezier}

A curva de Bezier, segundo LÉPINE; GUIBOULT; TRÉPANIER (2001), é uma curva polinomial expressada através da interpolação linear entre " $n$ " pontos, conhecidos como pontos de controle. O polinômio da curva de Bezier pode ser representado pela equação abaixo conforme LEIFSSON; KOZIEL 2015):

Eq.(1)

$$
B(t)=\sum_{k=1}^{m} \sum_{i=1}^{n} \frac{n !}{i !(n-1) !}(1-t(k))^{n-1} t(k)^{i} P(i)
$$

Onde $\mathrm{Pi}, \mathrm{i}=0,1, \ldots, \mathrm{n}$, são os pontos de controle, e " $\mathrm{t}$ " é uma matriz 1 x m com entradas de 0 a 1 . A Figura 5 abaixo ilustra uma curva de Bezier cúbica:

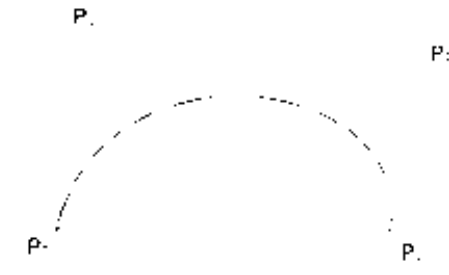

Figura 5: Demonstração de uma curva de Bezier. Fonte: LEPINE et al, 2001. 


\section{FORMULAÇÃO DO PROBLEMA}

$\mathrm{O}$ desenvolvimento deste estudo se baseia no artigo LEIFSSON; KOZIEL (2015) onde são realizadas simulações numéricas em software de CFD (Computational Fluid Dynamics) para avaliar a curvatura das paredes do bocal do túnel de vento.

\subsection{Dimensões do Túnel de Vento}

O bocal do túnel de vento estudado possui 2 metros de comprimento, seção de entrada com $2 \mathrm{~m}$ de altura por $2 \mathrm{~m}$ de largura, e seção de saída com $0,71 \mathrm{~m}$ por 0,71 $\mathrm{m}$. Formando assim, entrada e saída, ambas com seção quadrada de áreas de $4 \mathrm{~m}^{2}$ e $0,5041 \mathrm{~m}^{2}$ respectivamente. Desta forma a razão entre a área da saída e da entrada é, aproximadamente, de 1 para 8. A câmara de estabilização tem comprimento de $1,50 \mathrm{~m}$. O túnel possui 8 metros de comprimento total. Para alcançar uma velocidade de $20 \mathrm{~m} / \mathrm{s}$ na seção de testes, a vazão do fluxo deve ser aproximadamente $40.000 \mathrm{~m}^{3} / \mathrm{h}$. A Figura 6ilustra o túnel acima mencionado:

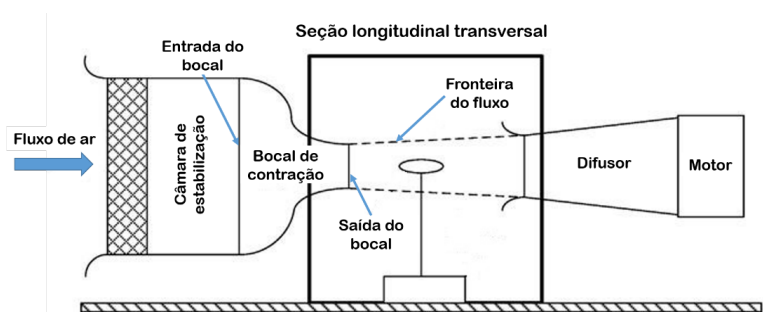

Figura 6: Representação do túnel de vento contruído por LEIFSSON et al.

Fonte: LEIFSSON et al, 2015 (adaptada).

\subsection{Desenvolvimento do Projeto}

Os testes foram desenvolvidos em um quarto do volume representado pelo desenho do corpo do bocal, conforme demonstrado na Figura abaixo. Segundo LEIFSSON; KOZIEL (2015), isso melhora o tempo computacional da simulação sem interferir no resultado final.

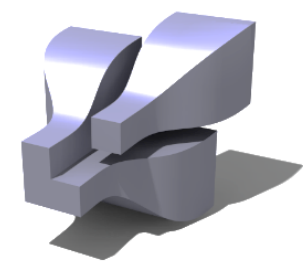

Figura 7: Imagem representando corte de $\frac{1}{4}$ do bocal.
Com a divisão do bocal, a área da entrada agora possui $1 \mathrm{~m}^{2}$ e a área da saída corresponde a $0,355 \mathrm{~m}$ de altura por $0,355 \mathrm{~m}$ de largura (aproximadamente 0,126 $m^{2}$ ), o comprimento permanece 2 metros. A curvatura da parede de contração é gerada através de 5 pontos descrevendo uma curva de Bezier (P1, P2, P3, P4, $\mathrm{P} 5)$ (Figura 8). Sabendo que a área da entrada não varia, o ponto $\mathrm{P} 1$, para todas as curvas, permanece sem variação de posição. Da mesma forma P5 que corresponde a aresta da saída do bocal tem sua posição fixa. Foi convencionado que o P3 está fixado no ponto central da reta que liga P1 e P5. Os pontos P2 e P4 variam apenas ao longo de $\mathrm{x}$. A Figura abaixo descreve como são dispostos os pontos em função do plano cartesiano.

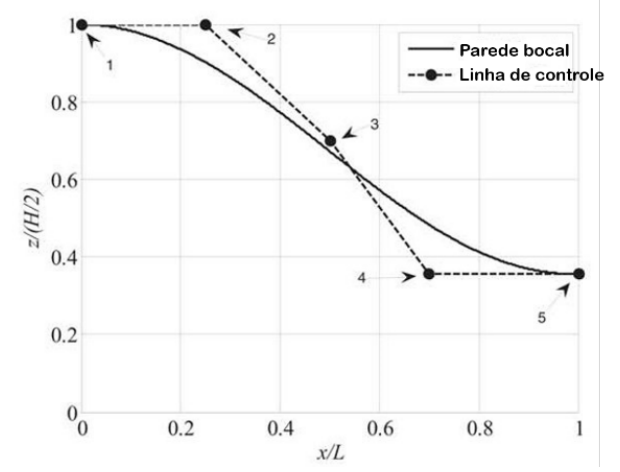

Figura 8: Pontos para construção das curvas de Bezier. Fonte: LEIFSSON et al, 2015 (adaptada).

Desta forma tem-se duas variáveis, $x_{2}$ e $x_{4}$. A distância de $x_{2}$ em relação a $x_{1}$ é a mesma de $x_{4}$ em relação a $x_{5}$, mudando apenas o sentido do deslocamento a cada curva. Os pontos P2 e P4 se deslocam $25 \mathrm{~cm}$ a cada curva. Foram desenhadas 5 curvas conforme demonstrado na Figura 9

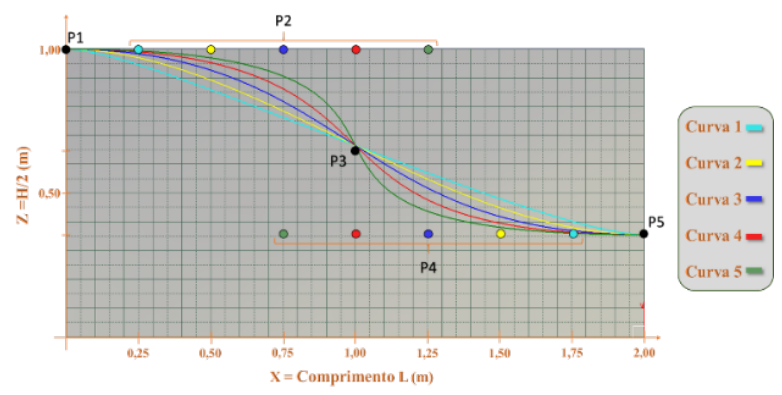

Figura 9: Pontos e curvas sugeridas.

A partir das curvas descritas acima, foram geradas 5 geometrias representando as respectivas situações analisadas no trabalho. 


\subsection{Modelo Computacional}

Uma simulação básica de CFD, em geral, é descrita em quatro passos: desenho da geometria; geração da malha para a geometria; solução numérica que rege a equação de fluxo do fluido; e processamento dos resultados.

\subsubsection{Equações numéricas}

Foi determinado fluxo sendo estável, tridimensional, incompressível e turbulento. As equações que regem o fluxo do fluido são determinadas pelas equações de Navie-Stokes. O fluido utilizado nas simulações é o ar, considerando uma temperatura de $25{ }^{\circ} \mathrm{C}$. Segundo F. Menter, os cálculos da turbulência devem ser desenvolvidos pelo Shear Stress Transport $(S S T)_{k-w}$.

\subsubsection{Malha computacional}

Conforme ilustrado na Figura 10, a contração é simétrica em relação ao plano xy e xz. Portanto, conforme mencionado na seção 4.2, o domínio desenvolvido é $\frac{1}{4}$ da geometria. A estrutura da malha é do tipo hexaédrica. A letra $\mathrm{N}$ representa o número de nós em cada eixo $(\mathrm{x}, \mathrm{y}, \mathrm{z})$, sendo representado respectivamente por $N_{x}, N_{y}$ e $N_{z}$, onde $N_{y}$ e $N_{z}$ são configurados igualmente. A malha foi desenvolvida para ser refinada próximo a parede para captar possíveis turbilhonamentos. A malha foi projetada no software ICEM da plataforma ANSYS 15. Foram elaboradas 4 malhas com refinos diferentes (malha grossa, malha média, malha fina, e malha extrafina) e realizado um estudo para determinar qual seria a melhor opção para os testes subsequentes.
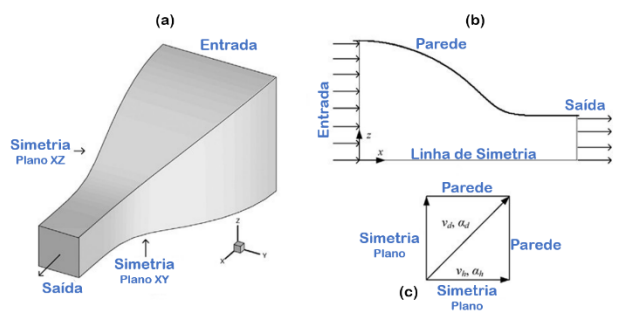

Figura 10: Definição dos eixos X, Y, e Z. Fonte: LEIFSSON et al, 2015 (adaptada).

\subsubsection{Solução numérica}

Através do SOLVER do CFX, a solução por volumes finitos (MALISKA, 2004) das equações de NavierStokes, utilizando os parâmetros pré-estabelecidos. O programa refaz os cálculos até que os resíduos sejam considerados desprezíveis.

\section{METODOLOGIA}

A metodologia utilizada no desenvolvimento deste estudo se baseia no artigo de (LEIFSSON; KOZIEL, 2015), aonde são definidas as fases conforme descrito na seção 4.3. O desenvolvimento da geometria baseando-se na curva de Bezier, de acordo com as descrições mencionadas na seção 4.2.

\subsection{Estudo de Malha}

Foi desenvolvida uma geometria de controle baseado no túnel de vento descrito na referência LEIFSSON; KOZIEL (2015). A partir desta geometria foram geradas 4 malhas de refino diferentes conforme Tabela 1 .

Tabela 1: Demonstrativo de refinamento das malhas

\begin{tabular}{cccccc}
\hline \multicolumn{5}{c}{ REFINO DE MALHA } \\
\hline Nós & Distri. & Grossa & Média & Fina & Extra Fina \\
\hline $\mathrm{N}_{x}$ & Unif. & 25 & 40 & 80 & 160 \\
$\mathrm{~N}_{y}$ & Geom. 2 & 15 & 40 & 60 & 120 \\
$\mathrm{~N}_{z}$ & Geom. 2 & 15 & 40 & 60 & 120 \\
\hline \multicolumn{7}{c}{ Total de Nós } & 5.625 & 64.000 & 288.000 & 2.304 .000
\end{tabular}

O estudo foi realizado usando os mesmos parâmetros de simulação para as quatro malhas e em seguida foram analisadas as variações da velocidade de cada malha próximo à aresta de intercessão das paredes na seção de saída. Conforme pode-se observar na Figura 11. a seção representada pela malha grossa não possui definição adequada para ilustrar a variação da velocidade nesta região. Em contrapartida, a malha de refinamento extra fina por possuir uma quantidade elevada nós, requer maior tempo de processamento computacional para solucionar as equações de NavierStokes. A malha média não capta adequadamente as velocidades no canto da geometria, desta forma a malha fina foi definida como padrão para as simulações nas curvas descritas na seção 4.2.

\subsection{Geometria}

Foram geradas 5 geometrias seguindo as descrições anteriores utilizando o software CATIA V5. Como demonstrativo a figura abaixo representa a geometria da curva 4.

\subsection{Geração das Malhas}

Segundo o estudo da seção 5.1 foram geradas as malhas para cada curva sugerida na seção 4.2 , aonde a Figura 12 apresenta a localização dos itens da malha (entrada, saída, paredes e simetrias). A distribuição da malha foi configurada para concentrar os nós próximos 


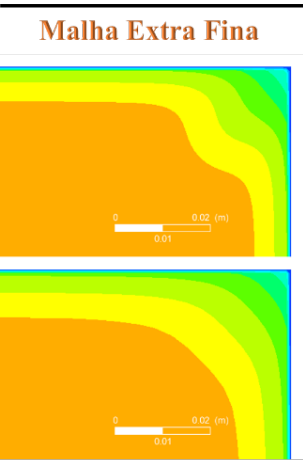

Malha Média

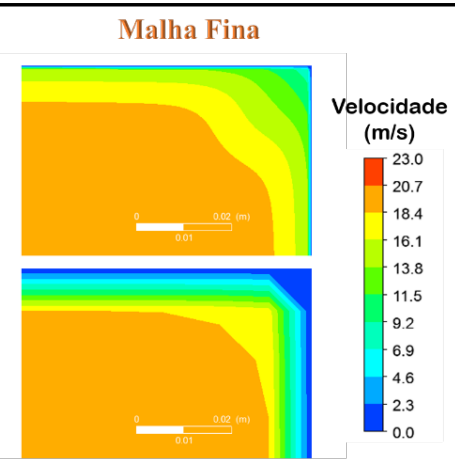

Malha Grossa
Figura 11: Comparativo de malhas.

as paredes de forma a garantir uma maior precisão nesta região.

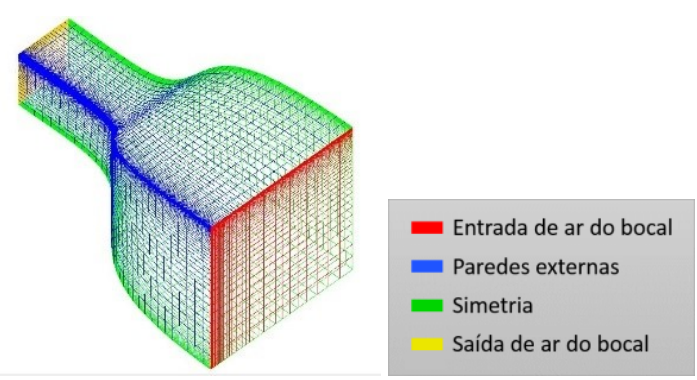

Figura 12: Representação da malha do bocal no ICEM.

\subsection{Configuração dos Parâmetros da Simulação}

Utilizando o CFX-PRE, deve-se criar um novo caso, em seguida importar a malha gerada no ICEM. Feito isso, deve ser criado um domínio para configurarmos as características do fluido utilizado nos testes e as equações para solução. Foi considerado o $\mathrm{Ar}$ a $25^{\circ} \mathrm{C}$ como fluido. A simulação é considerada isotérmica, pois não há nenhum trocador de calor. Conforme mencionado no item 4.3.1, foi definido como cálculo de turbilhonamento o Shear Stress Transport $(S S T)_{k-w}$. Ao concluir a formatação do domínio, foram configuradas as regiões de contorno conforme mencionado na seção 4.3.3: entrada com fluxo de massa; saída com pressão estática; paredes; e simetrias.

O CFX possui a ferramenta SOLVER para realizar os cálculos utilizando os parâmetros pré-estabelecidos. O programa refaz os cálculos até que os resíduos sejam considerados desprezíveis. No caso foi configurado para que o resíduo na ordem de $10^{-} 6$. Uma velocidade é prescrita como condição de contorno na seção de en- trada na forma de vazão mássica $(3,2885 \mathrm{~kg} / \mathrm{s})$ correspondente a vazão volumétrica descrita na seção 4.1. A condição de contorno na saída é definida como pressão ambiente. Simetria é a condição de contorno nas seções dos planos xy e xz. E por fim, é configurado nas superfícies de contração como parede.

Ao final dos cálculos realizados pelo SOLVER, o programa CFX-POST é aberto para que sejam gerados gráficos e imagens relevantes para demonstrar os resultados.

\section{RESULTADOS}

- Resultado estudo de malha Foram analisadas as velocidades de cada malha próximo à aresta das paredes nas superfícies de contração na seção de saída. Conforme foi demonstrado na Figura 11, a malha grossa apresenta uma distorção na aresta causada pelo baixo nível de refinamento. Em contrapartida a malha extrafina demanda de um tempo de processamento computacional extremamente elevado em relação às demais. Pode-se observar que a malha média não capta adequadamente as velocidades no canto da geometria em comparação à malha extra refinada. Desta forma a malha fina foi definida como padrão para as simulações nas curvas propostas do artigo.

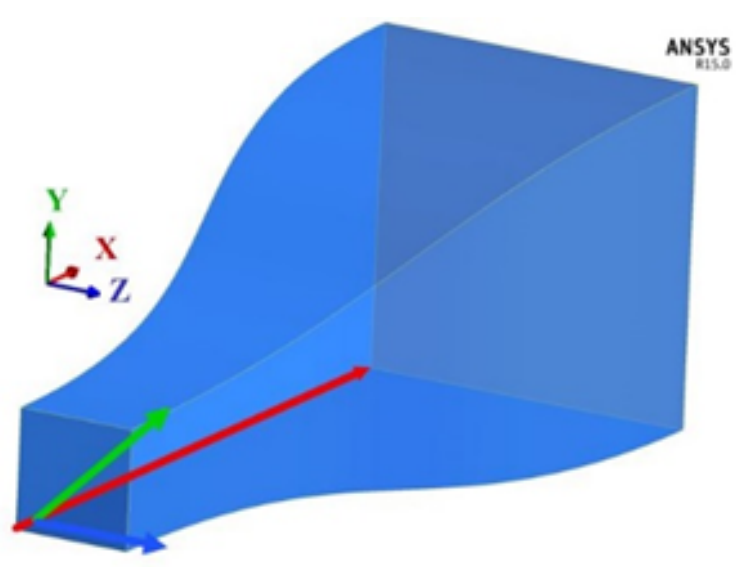

Figura 13: Eixos/linhas de referências.

- Resultados finais Após efetuar as simulações numéricas para as 5 curvas, foram gerados gráficos das velocidades em função de linhas referenciais ilustradas acima na Figura 13 . Avaliando numericamente os casos simulados podemos observar que quanto maior a contração da curva, mais rápido o ar atinge velocidades acima de $20 \mathrm{~m} / \mathrm{s}$, como podemos observar no Gráfico 1. Desta forma, podendo influenciar no comprimento necessário para estabilização do fluxo. Ainda no Grá- 
fico 1 , à $10 \mathrm{~cm}$ de distância da saída do bocal, pode ser observado que quanto maior a contratura maior a velocidade final do fluido, onde a curva 1 apresenta velocidade de aproximadamente $20 \mathrm{~m} / \mathrm{s}$ e a curva 5 apresenta velocidade próxima a $22 \mathrm{~m} / \mathrm{s}$. Tanto no Gráfico 2 quanto no Gráfico 3, foi identificada uma queda brusca na velocidade causada pelo atrito do fluido com as paredes do bocal. Entretanto podem ser observadas algumas diferenças entre as curvas. Embora a curva 5 demonstre maiores velocidades do centro até o início da camada limite, após a marca de $0,31 \mathrm{~m}$ a curva demonstra a menor velocidade exibida nos gráficos 2 e 3 .

Figura 14: Gráfico 1 - Eixo $X$, para $Y$ e $Z$ iguais a zero (linha vermelha da Figura 13).

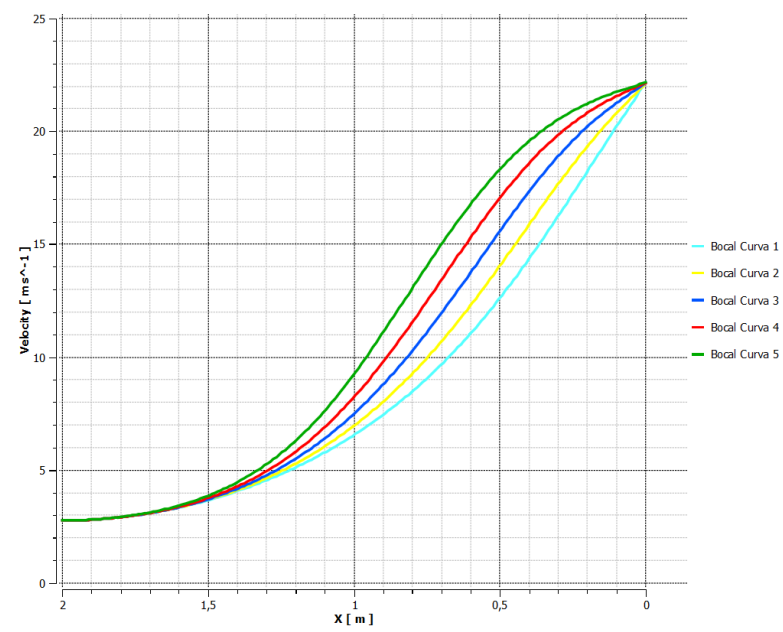

Fonte: Gerado automaticamente pelo CFX-Post - Ansys.

Figura 15: Gráfico 2 - Linha correspondente ao eixo $\mathrm{Z}$ à $10 \mathrm{~cm}$ da saída do bocal (linha azul).

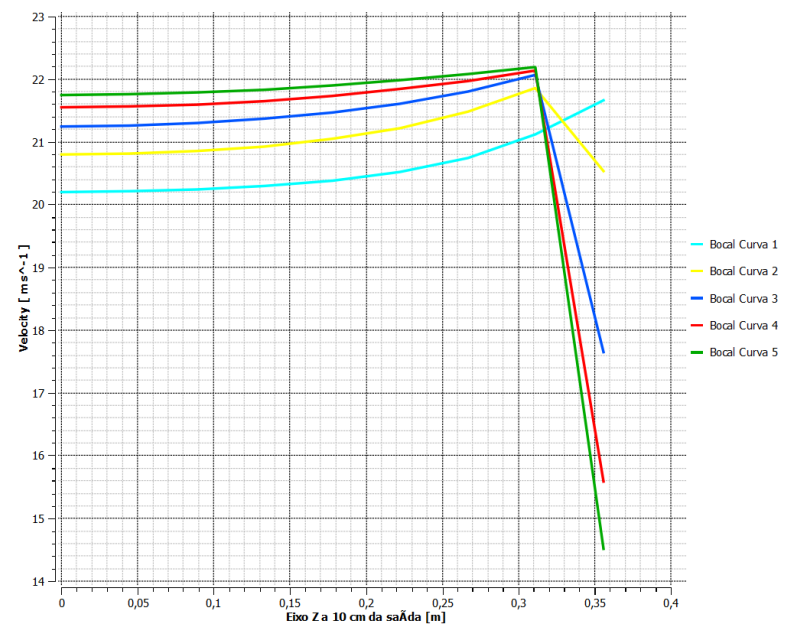

Fonte: Gerado automaticamente pelo CFX-Post - Ansys.
Figura 16: Gráfico 3 - Linha correspondente à diagonal YZ à $10 \mathrm{~cm}$ da saída do bocal (linha verde).

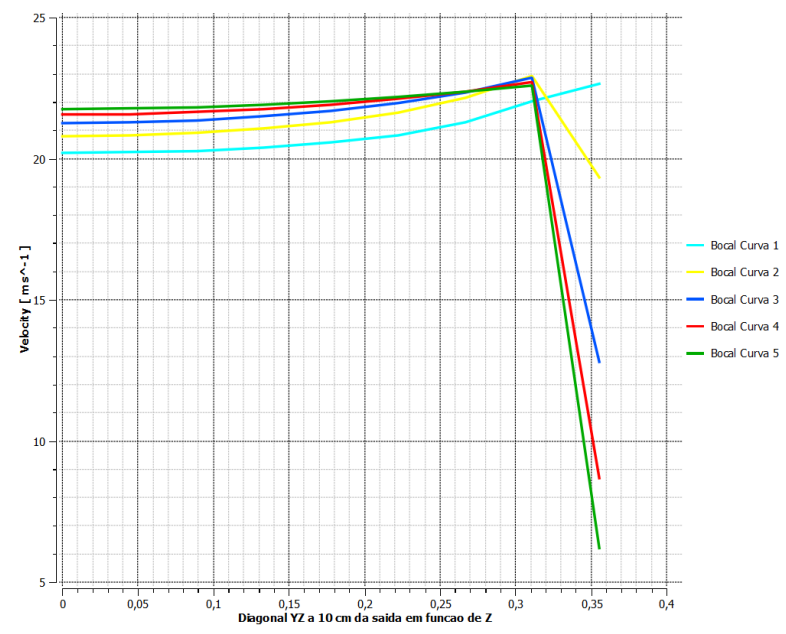

Fonte: Gerado automaticamente pelo CFX-Post - Ansys.

Para garantir que os resultados encontrados nos cálculos numéricos realizados pelo software sejam realistas, foram realizados testes comparativos com os resultados experimentais do túnel de vento mencionado por LEIFSSON et. al. (2015). O gráfico abaixo ilustra a comparação dos resultados obtidos através de simulações com um fluxo médio de $16,5 \mathrm{~m} / \mathrm{s}$ na seção de teste, aonde a variação de cores representa as velocidades obtidas através da simulação e as linhas brancas representam as velocidades experimentais.

Figura 17: Gráfico 4-Comparativo de velocidades experimentais com resultados simulados.

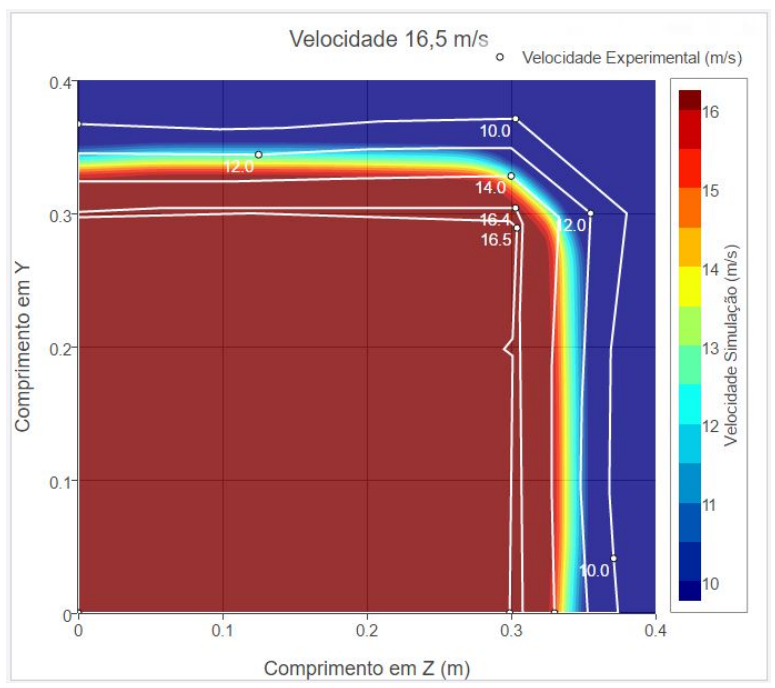

Fonte: Gerado automaticamente pelo CFX-Post - Ansys.

- Validação experimental 


\section{CONCLUSÃO}

Analisando o comparativo dos resultados apresentados em forma de gráficos, é possível observar que a curva 5 embora tenha apresentado melhor desempenho quanto a velocidade máxima alcançada ao longo do eixo $\mathrm{X}$ (aproximadamente $22 \mathrm{~m} / \mathrm{s}$ ), essa geometria apresenta uma camada limite maior que as demais, além de apresentar uma seção, no raio da curva de entrada, aonde a velocidade é nula. O fenômeno não é observado nas demais curvaturas. Portanto a curva 4 apresenta resultados mais homogêneos do que as demais, pois sua contração não gera tantas perdas e demonstra velocidades aproximadas em relação a curva 5 .

\section{REFERÊNCIAS}

ALÉ, J. A. V.; IZQUIERDO, R. C.; SIMIONI, G. d. S.; OLIVEIRA, C. P. Metodologia e resultados de calibração de anemômetro. V Congresso Brasileiro de Metrologia, Salvador, Bahia - Brasil, nov. 2009.

ARRIAS, L. L.; UEMURA, F. O.; FEIDEN, A.; MARRAFÃO, R. E.; GONçALVES, R. R. B. Projeto e construção de um túnel de vento subsônico de circuito aberto para utilização em laboratório didádico. TCC Universidade Estadual de Maringá-PR, 2008.

BARLOW, J. B.; RAE, W. H. J.; POPE, A. Low-Speed Wind Tunnel Testing. 3rd edition. ed. : John Wiley \& Sons, Inc, 1999.

GROFF, J.; ALÉ, J. V. Projeto de túnel de vento subsônico de circuito aberto. Rio Grande do Sul: PUC, 2000.

LEIFSSON, L.; KOZIEL, S. Simulation-driven design of low-speed wind tunnel contraction. Journal of Computational Science, 2015.

LÉPINE, J.; GUIBOULT, F; TRÉPANIER, J. Y. Optimized nonuniform rational b-spline geometrical representation for aerodynamic design of winds. AIAA Journal, v. 39, n. 11, p. 2033-2041, 2001.

MALISKA, C. R. Transferência de calor e mecânica dos fluidos computacional. : Livros Tecnicos e Cientificos, 2004.

MEASNET. Cup anemometer Calibration Procedure. 2009. Version 2.

MEHTA, R.; BRADSHAW, P. Tecnichal Notes: Design Rules for Small Low Speed Wind Tunnels. 1979.

\section{APÊNDICE}

Figura 18: Velocidades ao longo do bocal da Curva 1.

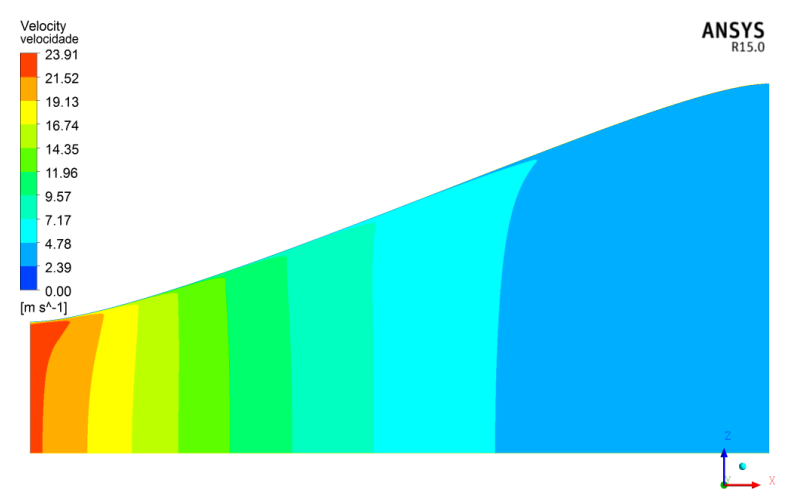

Figura 19: Velocidades ao longo do bocal da Curva 2.

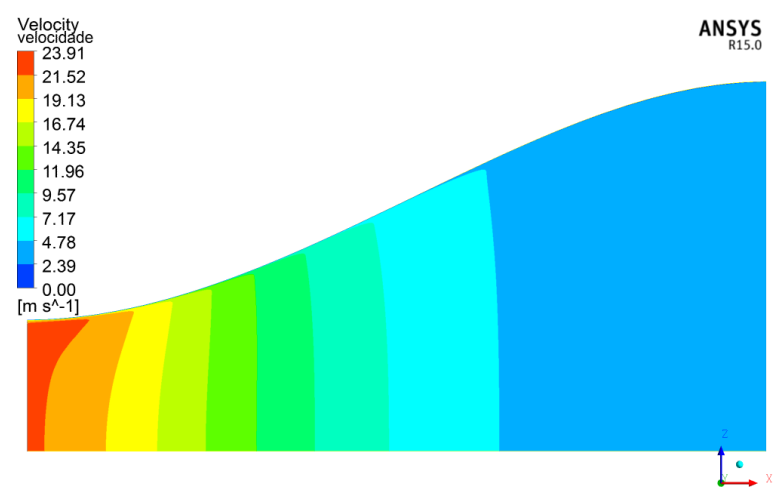

Figura 20: Velocidades ao longo do bocal da Curva 3.

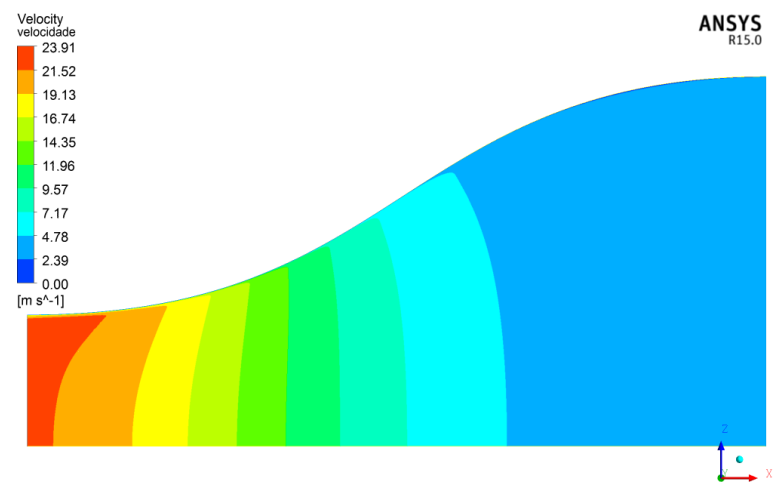


Figura 21: Velocidades ao longo do bocal da Curva 4.

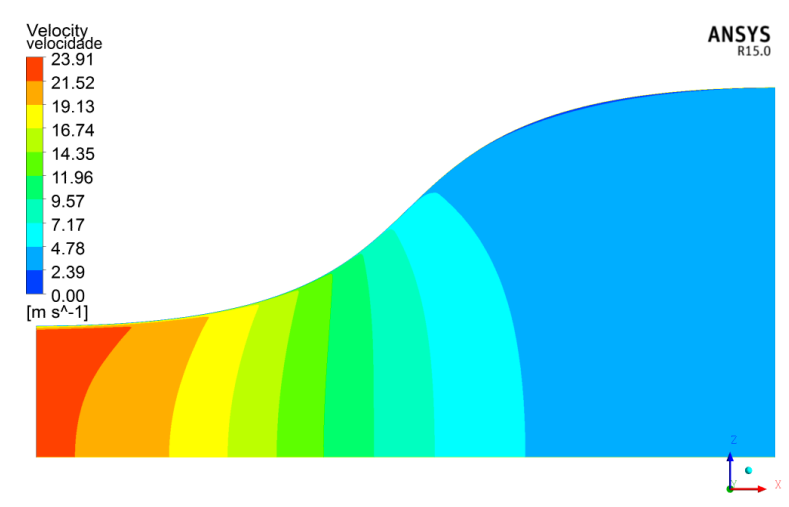

Figura 22: Velocidades ao longo do bocal da Curva 5.

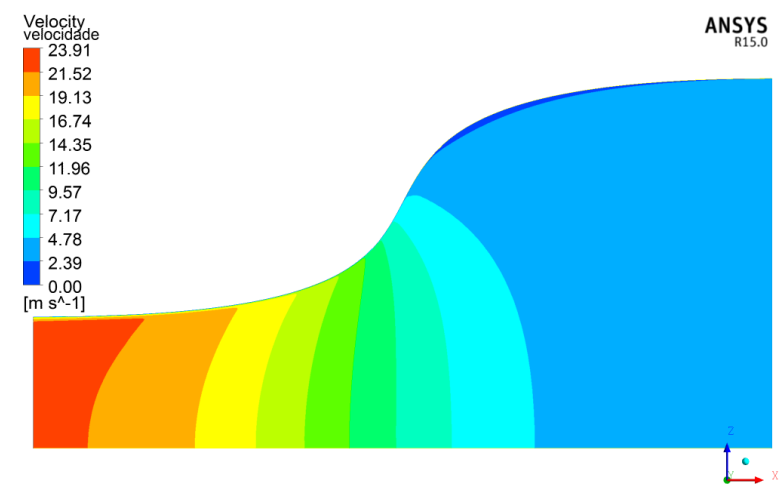

